ORNL/TM-2006/578

\title{
Optimization of the CMDFT code
}

\author{
July 2006
}

Xiaoguang Zhang and Paul Kent 


\title{
DOCUMENT AVAILABILITY
}

Reports produced after January 1, 1996, are generally available free via the U.S. Department of Energy (DOE) Information Bridge:

Web Site: http://www.osti.gov/bridge

Reports produced before January 1, 1996, may be purchased by members of the public from the following source:

National Technical Information Service

5285 Port Royal Road

Springfield, VA 22161

Telephone: 703-605-6000 (1-800-553-6847)

TDD: $703-487-4639$

Fax: 703-605-6900

E-mail: info@ntis.fedworld.gov

Web site: http://www.ntis.gov/support/ordernowabout.htm

Reports are available to DOE employees, DOE contractors, Energy Technology Data Exchange (ETDE), and International Nuclear Information System (INIS) representatives from the following sources:

Office of Scientific and Technical Information

P.O. Box 62

Oak Ridge, TN 37831

Telephone: $865-576-8401$

Fax: 865-576-5728

E-mail: reports@adonis.osti.gov

Web site: http://www.osti.gov/contact.html

\begin{abstract}
This report was prepared as an account of work sponsored by an agency of the United States Government. Neither the United States nor any agency thereof, nor any of their employees, makes any warranty, express or implied, or assumes any legal liability or responsibility for the accuracy, completeness, or usefulness of any information, apparatus, product, or process disclosed, or represents that its use would not infringe privately owned rights. Reference herein to any specific commercial product, process, or service by trade name, trademark, manufacturer, or otherwise, does not necessarily constitute or imply its endorsement, recommendation, or favoring by the United States Government or any agency thereof. The views and opinions of authors expressed herein do not necessarily state or reflect those of the United States Government or any agency thereof.
\end{abstract}


ORNL-1234

\title{
Optimization of the CMDFT code
}

Xiaoguang Zhang and Paul Kent

Date Published: July 2006

\author{
Prepared by \\ OAK RIDGE NATIONAL LABORATORY \\ P. O. Box 2008 \\ Oak Ridge, Tennessee 37831-6285 \\ managed by \\ UT-Battelle, LLC \\ for the \\ U. S. DEPARTMENT OF ENERGY \\ under contract DE-AC05-00OR22725
}




\section{Contents}

List of Figures

List of Tables $\quad$ v

Acronyms

Executive Summary vii

1 Introduction 1

2 Code efficiency before optimization 1

3 Optimization of bdvs 2

4 Optimization of the FFT routines 6

4.1 Setup ............................ 6

4.2 Computation of FFT . . . . . . . . . . . . . . 6

4.3 Data dependency in wave function storage . . . . . . . . . 7

4.4 New load-balancing algorithm . . . . . . . . . . . . 7

4.5 Unresolved problem . . . . . . . . . . . . . . 8

5 Summary of the speedup $\quad 8$ 


\section{List of Figures}

1 Scaling of CMDFT with number of processors before optimization. 2

2 Execution times of bdvs and gtorf $\mathrm{ft}$ as a function of the block size nwfg. . . . . . . . . . . . . . .

3 The three-dimensional FFT calculation is done as three sets of onedimensional FFT's. (a) The sphere of original reciprocal vectors; (b) After FFT along $x$; (c) Global transpose from (b), this step require the most communications; (d) After FFT along $y$; (e) Global transpose from (d), this step requires much less communication than from (b) to (c); (f) After FFT along $z$. Source: Andrew Canning, LBNL . . . . . . . . . . . . . . . . 


\section{List of Tables}

1 Efficiencies of top CMDFT routines before optimization for a 24 atom cluster test case using 32 nodes on Jaguar. . . . . . . . . . .

2 Efficiencies of top CMDFT routines after optimization for a 24 atom cluster test case using 32 nodes on Jaguar. . . . . . . . . . . 


\section{Acronyms}

BOMD: Born-Oppenheimer Molecular Dynamics

DFT: Density Functional Theory

FFT: Fast Fourier Transformation

SCF: Self-Consistent Field 


\section{Executive Summary}

This report outlines the optimization of the CMDFT code by Xiaoguang Zhang during June-July 2006. The overall improvement in speed is nearly 40\%. Possible further optimizations are also discussed. 


\section{Introduction}

The Born-Oppenheimer molecular dynamics (BOMD) method is a variant of the first-principles Car-Parrinello molecular dynamics. The BOMD method treats classically the nuclei motions and quantum mechanically the electrons using the density functional theory (DFT). The electronic structure is converged at every nuclei conformation. The Georgia Tech CMDFT code implements the BOMD method with a pseudopotential plane-wave basis. The detailed description of the method is given in [1].

The CMDFT code is written in f90, and uses MPI for communications. Here we summarize recent efforts in optimizing of the CMDFT code. All the tests are run on Jaguar.

\section{Code efficiency before optimization}

The test case used in this report is a nanocluster of 24 cobalt atoms. The reciprocal space grid is chosen as $\mathrm{nx}=\mathrm{ny}=128$, and $\mathrm{nz}=160$. The number of wave functions used in the calculation is nwfu $=240$. The calculation is stopped after $10 \mathrm{SCF}$ iterations, before convergence is reached.

As shown in Table 1, the overall efficiency of the CMDFT code is very low, reaching $2.5 \times 10^{9}$ FLOPS which is only $16 \%$ of peak floating point execution speed. This compares poorly to an average code that predominantly uses LAPACK routines. The expected performance for such a code is typically around $50 \%$ of peak. Therefore there seems to be a significant room for improvement.

Table 1: Efficiencies of top CMDFT routines before optimization for a 24 atom cluster test case using 32 nodes on Jaguar.

$\begin{array}{cccc}\text { Subroutine } & \text { Time }(\mathrm{sec}) & \text { Time\% } & \text { \% peak FLOPS } \\ \text { bdvs } & 239.28 & 37.0 & 17.5 \\ \text { gtorfft } & 152.23 & 23.5 & 11.7 \\ \text { rtogfft } & 116.62 & 18.0 & 13.1 \\ \text { hartree } & 34.15 & 5.3 & 36.7 \\ \text { ddendr } & 28.94 & 4.5 & 32.9 \\ \text { fftsetup } & 27.13 & 4.2 & 35.1 \\ \text { hwf } & 23.10 & 3.6 & 4.3 \\ \ldots & & & \\ \text { Total } & 676.38 & 100 & 16.3\end{array}$

The top three routines, bdvs, gtorfft, and rtogfft, are all involved in the 
process of finding the eigenenergies and the corresponding wave functions. This process requires the computation of the Hamiltonian matrix $H_{i j}=\left\langle\psi_{i}|H| \psi_{j}\right\rangle$ and the overlap matrix $S_{i j}=\left\langle\psi_{i} \mid \psi_{j}\right\rangle$, where $\psi_{i}$ are the trial wave functions. The trial wave functions $\psi_{i}$ are stored in terms of its expansion in plane waves, indexed with the reciprocal wave vectors. However, the vectors $H\left|\psi_{i}\right\rangle$ are computed in real space. So that a Fourier transform of $\psi_{i}$ is performed in gtorfft before the calculation. After the calculation a back Fourier transform is performed in rtogfft. At the end, the inner products $\left\langle\psi_{i}|H| \psi_{j}\right\rangle$ and $\left\langle\psi_{i} \mid \psi_{j}\right\rangle$ are computed in bdvs.

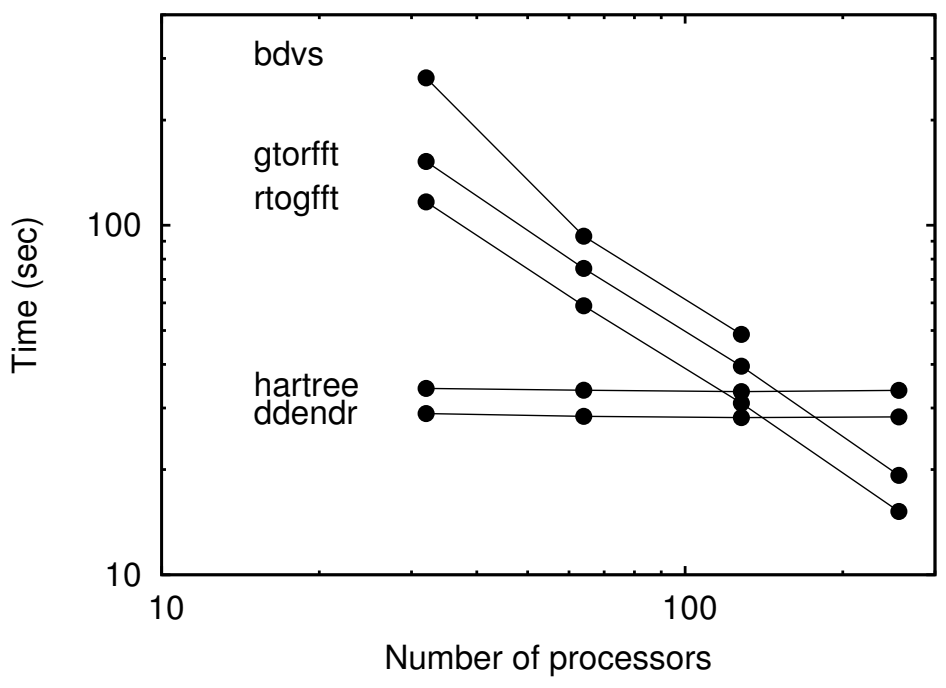

Figure 1: Scaling of CMDFT with number of processors before optimization.

The scaling of the top routines with the number of processors, on the other hand, shows very good linear scaling, as shown in Fig. 1. The flat performance of the two routines, hartree and ddendr, are due to the initial setup of the FFT in both routines. This will be discussed later along with the FFT optimization.

\section{Optimization of bdvs}

In bdvs, two matrices, $H_{i j}$ and $S_{i j}$ are computed by forming the inner products $\left\langle\psi_{i}|H| \psi_{j}\right\rangle$ and $\left\langle\psi_{i} \mid \psi_{j}\right\rangle$. Because both matrices are symmetric, only half of the matrix elements plus the diagonal ones are needed. This should save the computation time by about half. Unfortunately, to achieve this savings it prevents the usage of high level LAPACK routines. The original code uses ddot which results in a 
low efficiency of $17 \%$.

My first optimization attempt replaced all ddot calls with dgemv calls. Although this removes one explicit layer of do-loops, it made little difference in performance. One may instead forgo the factor of two savings and compute all elements, thus allowing the use of the highly efficient dgemm calls. The hope is that doubling in the number of computations will be more than compensated by the increase in efficiency.

In fact, we do not need to compute all elements to allow the use of the dgemm calls. The loop over the number of trial wave functions outside the ddot calls is divided into blocks of size nwf $\mathrm{g}$. This was originally designed to improve the efficiency of message passing during the FFT calculations. Typical values of nwf $g$ is between 10 and 100. Thus if we compute the matrix elements for each block of nwf $g$ wave functions together, filling all nwfg rows up to the largest size needed within the block, then dgemm can be used with a minimal increase in the number of elements computed. In Fig. 2 we show the results of this change. One can see that the execution time of bdvs is dramatically reduced as a function of nwf $g$, reflecting the improved efficiency due to better cache management by using dgemm. On the other hand, the execution time of gtorfft changes little with nwfg, allowing more flexibility with the choice of nwf $g$.

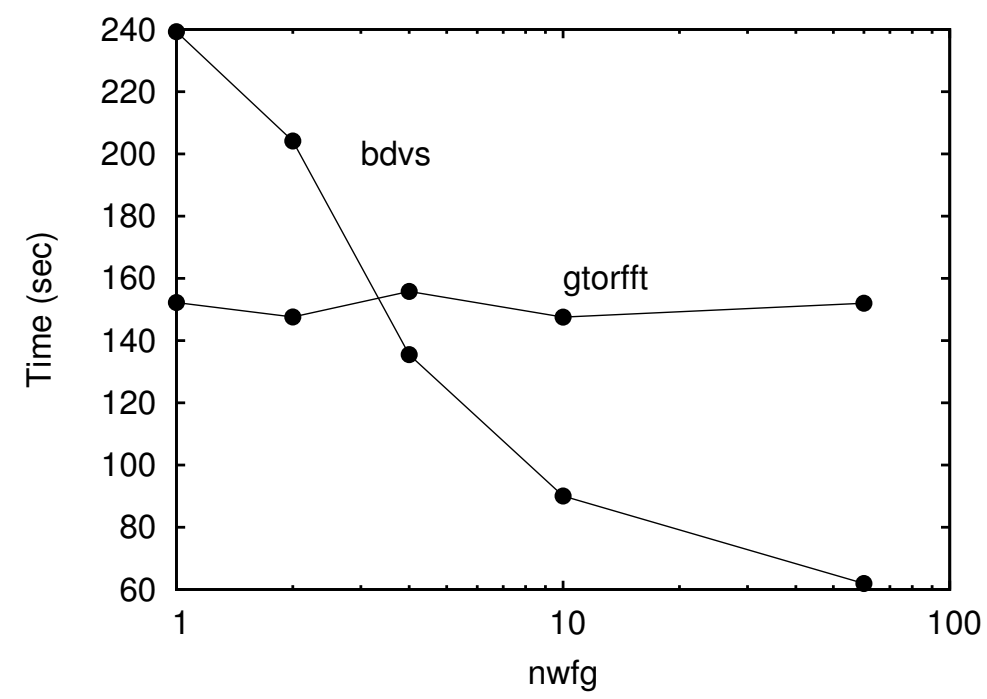

Figure 2: Execution times of bdvs and gtorfft as a function of the block size nwfg.

The changes are demonstrated in the following code example. Before the 
change,

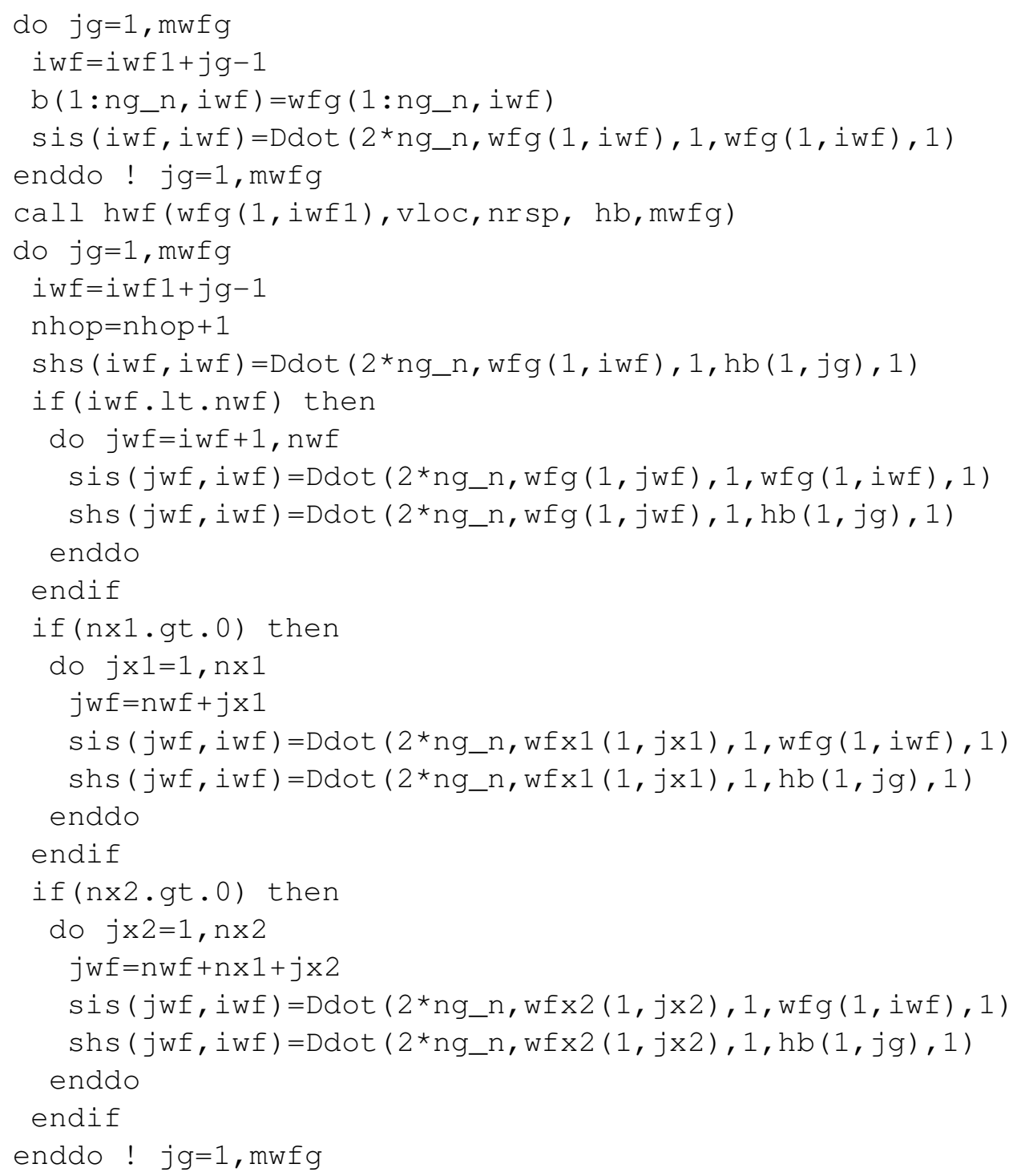

Compared to the code after the change,

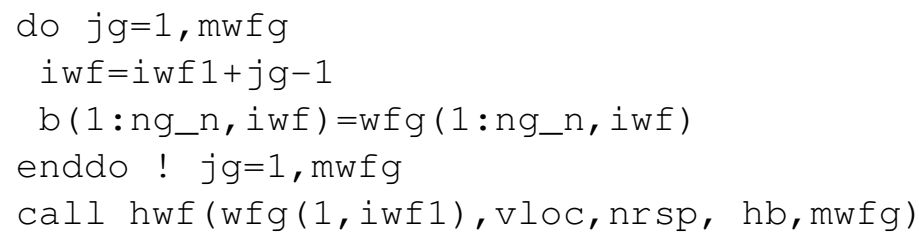




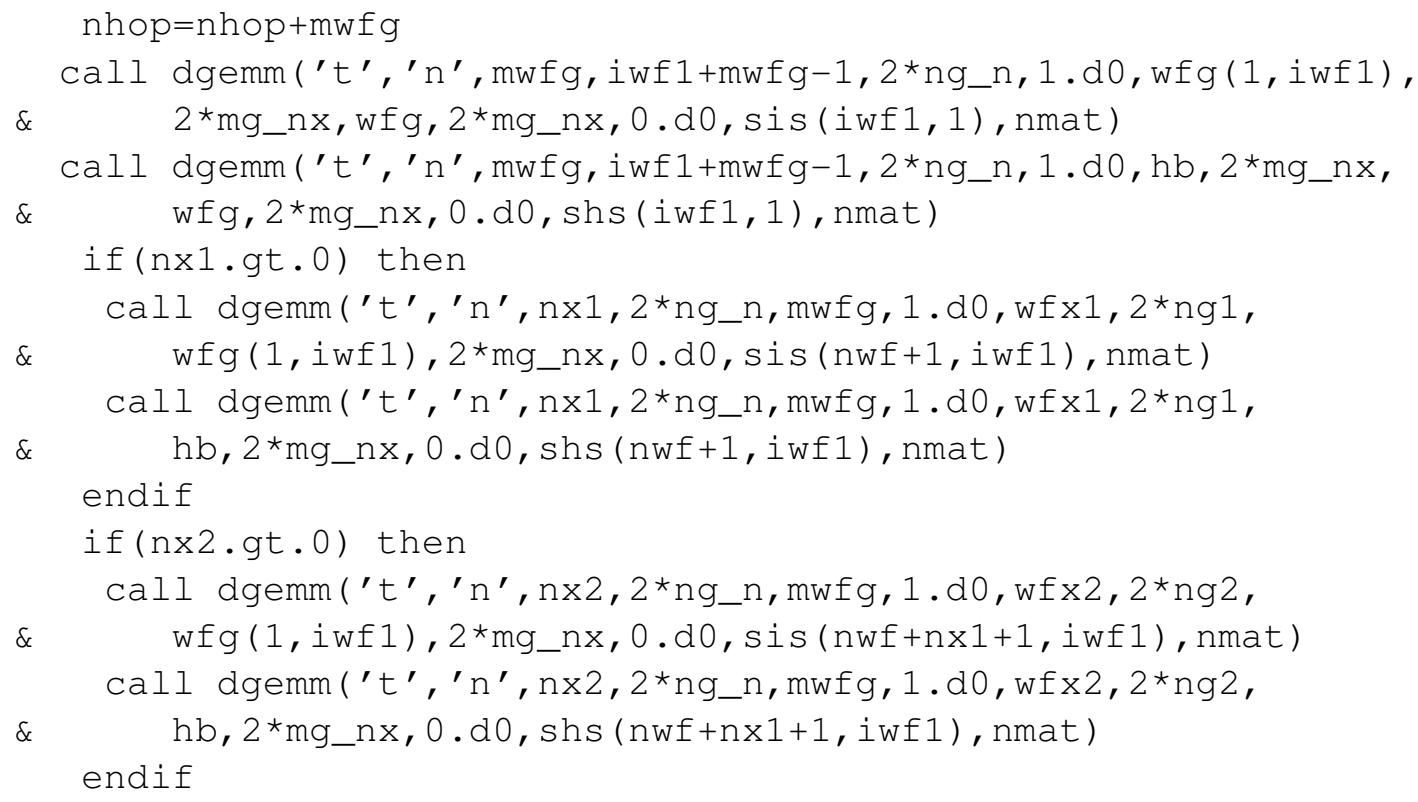

Similar changes are also made in other parts of bdvs. The final optimized version of bdvs yields a four-fold improvement in speed, reaching $71 \%$ peak performance, as shown in Table 2.

Table 2: Efficiencies of top CMDFT routines after optimization for a 24 atom cluster test case using 32 nodes on Jaguar.

$\begin{array}{cccc}\text { Subroutine } & \text { Time }(\mathrm{sec}) & \text { Time\% } & \text { \% peak FLOPS } \\ \text { gtorfft } & 125.49 & 30.1 & 14.2 \\ \text { rtogfft } & 103.47 & 24.9 & 14.7 \\ \text { fftsetup } & 75.69 & 18.2 & 36.4 \\ \text { bdvs } & 65.54 & 15.7 & 71.2 \\ \text { hwf } & 23.35 & 5.6 & 4.3 \\ \quad \ldots & & & \\ \text { hartree } & 0.92 & 0.2 & 12.9 \\ \text { ddendr } & 0.82 & 0.2 & 6.9 \\ \quad \ldots & & & \\ \text { Total } & 416.26 & 100 & 26.3\end{array}$




\section{Optimization of the FFT routines}

\subsection{Setup}

In the original code, FFT setup is done in three places, fftsetup for the wave function FFT, hartree for the Coulomb energy, and ddendr for charge density. As evident in the flat scaling of the execution time as a function of the number of nodes in Fig. 1, both hartree and ddendr are dominated by the setup of the FFT. Our first step in optimization of the FFT, is to merge the setup parts of both routines into fftsetup. This step cleans up the code structure significantly, while streamlines operation by removing duplicate parts. Table 2 shows the benefit of this change. Both hartree and ddendr now takes insignificant amount of time. The execution time of $\mathrm{ft}$ setup, although longer than before, is still much less than the sum of execution times of all three routines before the change.

\subsection{Computation of FFT}

The main compute routines for FFT include the forward FFT routine gtorfft, and the backward FFT routine rtogfft. The two FTT routines have very poor floating point efficiencies in the low teens. Because $f \mathrm{ftsetup}$ spends most of its time on trial runs of the FFT calculations, we can use its floating point efficiency, about $36 \%$, as the target efficiency for the two FFT routines. This would represent a factor of three improvement in efficiency.

In both gtorfft and rtogfft, most of the time is spent on message passing between nodes and on transposing arrays to prepare for the FFT calls. Both can be improved by optimizing the distribution of the wave functions on the nodes.

The total number of plane waves is approximately $\mathrm{nx} \times \mathrm{ny} \times \mathrm{nz}$. This is often a much larger number than the number of processors. Thus each processor is distributed several plane waves. In order to balance the load and memory requirement, the plane waves are not stored in sequence. Instead, wave vectors that share the same $y$ and $z$ indices are grouped into a column. Columns may have difference size (number of $x$ indices) due to the truncation of the reciprocal vectors $(x, y, z)$ at a spherical surface. Thus, these columns are first sorted according to their sizes, then distributed to the nodes according to a load-balanced algorithm.

This load-balanced distribution of the wave vectors significantly complicates the FFT calculation. The first FFT, along the $x$ direction, is straightforward. Then, before the FFT along $y$, wave vectors need to be redistributed between the nodes, creating significant amount of message passing, and then the arrays need to be transposed to move the $y$ index to the front. The same process is then repeated for $z$ (see Fig. 3). 
The key to optimization, is to design a distribution algorithm, that minimizes the message passing and transposing for the $y$ and $z$ directions, while keeping the load balanced. Before we discuss that, we need first to discuss some relevant data dependencies.

\subsection{Data dependency in wave function storage}

In the CMDFT code, assumptions on the order of the plane wave arrays are used in many subroutines. These assumptions are used to truncate the arrays, to throw away wave vectors not needed due to symmetry, and to map wave functions between $x$ and $-x$ reciprocal vectors. Such strong data dependency must be removed before any change in load-balancing algorithm can be implemented.

Concurrent changes are made to both wfgsetup and wfgsetup 1 in order to remove this data dependency. Indexing arrays i 1 ton, i2ton, i3ton, ntok 1 , ntok2, ntok3, n1_inv, and n2_inv, are first generated using a particular order appropriately chosen (this choice will be discussed in more detail below). Then these arrays are used to produce the reciprocal vectors and to perform all other tasks. In this manner, only the generation of the indexing arrays depend on the particular algorithm, while the rest of code is completely independent.

\subsection{New load-balancing algorithm}

In the original algorithm implemented in wfgsetup and wfgsetup 1, the wave vectors are loaded onto each node considering two factors, the size of the column being loaded, and the total size of the array already on the node. By loading the columns in the decreasing order in size, and onto the node with the least amount of data, an approximate load-balancing is achieved. The new algorithm takes into an additional consideration, the $(y, z)$ coordinate of the column being loaded. The first attempt is to load the column onto a node that already has larger columns that shares an identical $z$ coordinate. By maximizing the number of columns that share the same $z$ coordinate on a single node, we minimize the amount of message passing before the FFT in the $y$ direction.

A related change is made in $\mathrm{f} f \mathrm{tset} u p$ which distributes $z$ slabs onto different nodes. Here the order of the loop over $z$ is changed to maximize the possibility that two slabs with the same $z$ can stay on the same node.

The improved result is shown in Table 2. We see that the speed of gtorfft is improved by about $18 \%$, and rtogfft by about $12 \%$. 


\subsection{Unresolved problem}

Although the algorithm change discussed above is successful, some of the other alternative ordering of the columns produced incorrect answers. This is likely caused by hidden data dependency not yet discovered. Further work is needed to find and remove this dependency.

Once the data structure is completely isolated, we are free to test more loadbalancing algorithms to find the optimal choice.

\section{Summary of the speedup}

After all the changes, the total speed of the CMDFT code is improved by nearly $40 \%$. The floating point efficiency improved from $16 \%$ to $26 \%$ of the peak. At this point, the execution time is dominated by the FFT. Despite the improvement made on the load-balancing algorithm, both FFT routines still have poor $14 \%$ efficiency. Eliminating the hidden data dependency will allow the implementation of a more localized load-balancing scheme to further improve the FFT efficiency. However, staying with three separate one-dimensional FFT's will limit the potential for significant further speed improvement. A change in the algorithm on a higher level to reduce the number of FFT calculations in each SCF iteration can both boost floating point efficiency and improve scaling.

\section{References}

[1] Robert N. Barnett and Uzi Landman, "Born-Oppenheimer moleculardynamics simulations of finite systems: Structure and dynamics of $\left(\mathrm{H}_{2} \mathrm{O}\right)_{2}$," Phys. Rev. B 48, 2081-2097 (1993). 
$(a)$

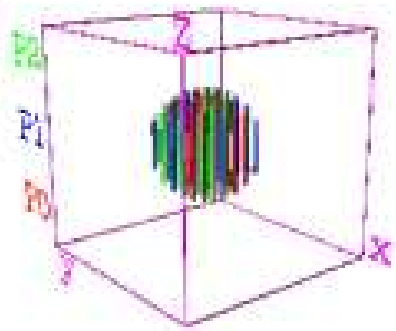

(c)

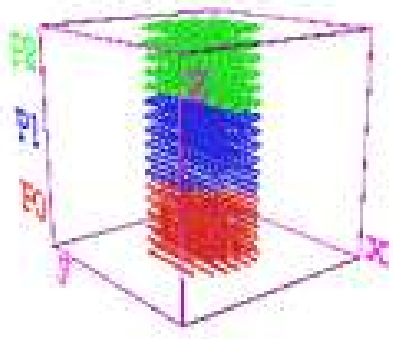

(ब)

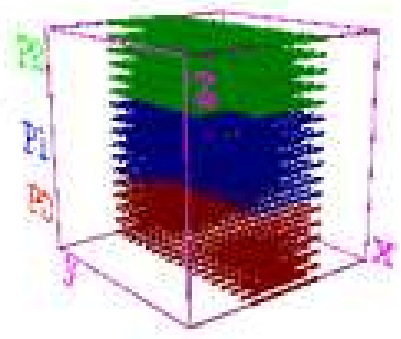

(b)

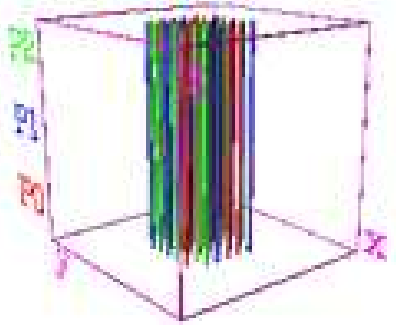

(d)

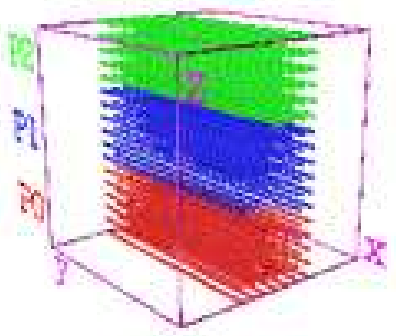

(9)

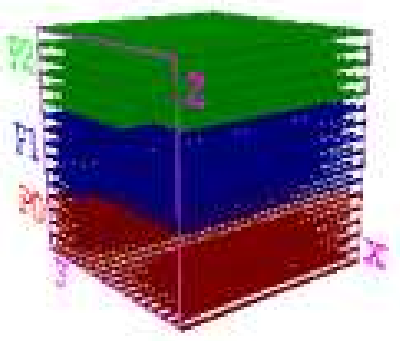

Figure 3: The three-dimensional FFT calculation is done as three sets of onedimensional FFT's. (a) The sphere of original reciprocal vectors; (b) After FFT along $x$; (c) Global transpose from (b), this step require the most communications; (d) After FFT along $y$; (e) Global transpose from (d), this step requires much less communication than from (b) to (c); (f) After FFT along $z$. Source: Andrew Canning, LBNL 